\title{
Risk factors for linezolid-induced thrombocytopenia in adult inpatients
}

\author{
Xiaonian $\mathrm{Han}^{1} \cdot$ Jinping Wang ${ }^{1} \cdot \mathrm{Xin} \mathrm{Zan}^{1} \cdot$ Lirong Peng $^{1} \cdot$ Xiaojing Nie $^{1} \mathbb{C}$
}

Received: 1 August 2021 / Accepted: 18 October 2021 / Published online: 3 November 2021

(c) The Author(s), under exclusive licence to Springer Nature Switzerland AG 2021

\begin{abstract}
Background Previous reports about risk factors for linezolid-induced thrombocytopenia have been insufficient, often due to the variability in study design and population, and some factors have not yet been studied. Aim The aims of this study are to determine potential risk factors for linezolid-induced thrombocytopenia, and to analyze the influencing factors of different thrombocytopenia definitions. Method This retrospective study involved patients who were administered intravenous linezolid for $\geq 1$ day between January 1, 2015 and January 1, 2021. Their demographic and clinical data were extracted from electronic medical records. Thrombocytopenia was defined as: (1)thrombocytopenia with platelet count $<100 \times 10^{9} / \mathrm{L}$ and a decrease in $25 \%$ or more from baseline of the platelet count (criterion 1); (2thrombocytopenia due to a platelet count drop decrease of $25 \%$ or more from baseline (criterion 2). Risk factors were determined via binary logistic regression analysis. Results This study included 320 patients. Binary logistic regression analysis indicated that baseline platelet count $(p<0.001)$, linezolid therapy duration $(p=0.001)$ and shock (patients require vasoactive medications) $(p=0.019)$ were independent risk factors for criterion-1 thrombocytopenia, while linezolid therapy duration $(p<0.001)$ and shock $(p=0.015)$ were independent risk factors for criterion- 2 thrombocytopenia. There was also a significant correlation between shock and early-onset thrombocytopenia ( $p=0.005$ and 0.019 for criterion 1 and criterion 2, respectively). Conclusion Linezolid therapy duration and shock were common causes of different thrombocytopenia definitions; shock was correlated with early-onset thrombocytopenia. Platelet count should be monitored during linezolid therapy especially during long-duration therapy and in shock patients.
\end{abstract}

Keywords Linezolid $\cdot$ Thrombocytopenia $\cdot$ Risk factor $\cdot$ Shock

\section{Impacts on practice}

1. Linezolid therapy duration and shock patients requiring vasoactive drugs were risk factors of linezolid-induced thrombocytopenia.

2. Shock was correlated with early-onset thrombocytopenia.

3. Platelet count should be monitored during linezolid therapy especially during long-duration linezolid therapy and in shock patients.

Xiaojing Nie

niexiaojingwell@163.com

1 Department of Pharmacy, Xi' an Central Hospital, Houzaimen No.185, North Street, Xian, Shaanxi, 710003, China

\section{Introduction}

Linezolid, a new oxazolidinone antimicrobial drug, has been used against Gram-positive bacterial infections such as methicillin-resistant staphylococci and vancomycin-resistant enterococci [1]. The antimicrobial mechanism of linezolid prevents the formation of a 70S initiation complex by binding to a site near the central region of domain $\mathrm{V}$ of the $23 \mathrm{~S}$ ribosomal RNA in the 50S subunit, which includes fMet transfer RNA, messenger RNA and the two ribosomal subunits [2, 3]. Cross-resistance does not occur, since no other known antimicrobial agent inhibits this process. Recent studies have indicated that linezolid has a positive effect on Mycobacterium tuberculosis (TB), and has been suggested to provide statistically significant treatment benefits to adult patients exhibiting extensive drug resistance [4]. In 2016, the World Health Organization recommended linezolid as a core second-line medicine in multidrug resistant tuberculosis (MDR-TB) regimen [5]. Linezolid has became widely used 
alongside the increasing prevalence of multidrug-resistant bacteria.

Linezolid is well tolerated, but myelosuppression in the form of thrombocytopenia and anaemia is a significant adverse drug reaction, of which thrombocytopenia has the highest risk. Reported risk factors of linezolid-induced thrombocytopenia included linezolid therapy duration [6-10], decreased creatinine clearance [6-13], baseline platelet count $[6,8,11,14,15]$, concomitant drugs with thrombocytopenic adverse effects $[8,11,13]$, chronic disease complications [10] and low body weight $[8,15]$. However, the studies of these risk factors were insufficient and often limited due to variability in study design, patient population and thrombocytopenia definitions. In clinical practice, critically ill patients, especially shock patients requiring vasoactive medications suffered severe thrombocytopenia shortly after linezolid treatment. So these further potential risk factors for linezolid-induced thrombocytopenia should be investigated.

\section{Aim of the study}

The aims of this study are to determine potential risk factors for linezolid-induced thrombocytopenia, including early onset thrombocytopenia, and to analyze the influencing factors of different thrombocytopenia definitions.

\section{Ethics approval}

This study was approved by the medical ethics committee of Xi' an Central Hospital (No. LW-2021-011). Data was de-identified prior to analysis so patient consent was not required.

\section{Method}

\section{Study design and population}

This retrospective study was conducted on patients from $\mathrm{Xi}$ ' an Central Hospital, which is a 1,000-bed tertiary-care teaching hospital in China. The population consisted of hospitalized patients aged $\geq 18$ years who received intravenous linezolid ( $0.6 \mathrm{~g}, \mathrm{q} 12 \mathrm{~h})$ for $\geq 1$ day between January 1, 2015 and January 1, 2021. Patients were excluded if they had haemato-oncologic diseases, myelosuppression, chemotherapy, COVID-19 or other viral disease, or incomplete baseline data.

\section{Data collection and thrombocytopenia definition}

Demographic and clinical data of each patient were collected, including sex, age, admission department, primary diagnosis, comorbidities, hospitalization days, the dose of linezolid and the frequency used, concurrent medications and baseline laboratory data prior to the first day of linezolid treatment including creatinine, total bilirubin, alanine aminotransferase, glutamic oxaloacetic transaminase, total protein, serum albumin, platelet count, haemoglobin level, intensive care, shock and linezolid treatment duration. Platelet count data were also collected after day 1 of linezolid treatment until discharge.

Thrombocytopenia was defined as two different criteria: (1)thrombocytopenia with platelet count $<100 \times 10^{9} / \mathrm{L}$ and a decrease in $25 \%$ or more from baseline of the platelet count (criterion 1); (2)thrombocytopenia due to a platelet count drop decrease of $25 \%$ or more from baseline (criterion 2). Early-onset thrombocytopenia was defined as thrombocytopenia occurrence within 6 days of linezolid treatment initiation.

\section{Statistical analyses}

All analyses were conducted using IBM SPSS (version 23.0). Continuous data were expressed as mean \pm SD values; and categorical data were expressed as frequencies. The differences in thrombocytopenia frequency as a function of sociodemographic characteristics were examined using independent-samples t-tests and Pearson's chi-square test. Binary logistic regression model was used to evaluate the factors associated with thrombocytopenia. The cumulative incidence of thrombocytopenia was compared between patients with different clinical conditions using a log-rank test and presented as Kaplan-Meier curves. Two-tailed probability values of $p<0.05$ were considered statistically significant.

\section{Results}

\section{Demographics and clinical characteristics}

Table 1 listed the characteristics of the study population. After excluding 116 participants with haemato-oncologic diseases, myelosuppression, chemotherapy, incomplete clinical data or aged $<18$ years, 320 patients who received intravenous linezolid treatment between January 1, 2015 and January 1, 2021 were included in this study. The age of the patients was $69.67 \pm 16.39$ years, and $69.06 \%$ of the population was male. The duration of hospitalization 
Table 1 Demographic and clinical characteristics of the study patients $(n=320)$

\begin{tabular}{|c|c|}
\hline Characteristics & Value \\
\hline Age (years), mean \pm SD & $69.67 \pm 16.39$ \\
\hline Male, n (\%) & $221(69.06 \%)$ \\
\hline Hospitalization days, mean \pm SD & $24.57 \pm 15.58$ \\
\hline Linezolid therapy duration, days, mean $\pm \mathrm{SD}$ & $7.40 \pm 5.75$ \\
\hline \multicolumn{2}{|l|}{ Creatinine clearance, $\mathrm{mL} / \mathrm{min}$} \\
\hline$\geq 60 \mathrm{ml} / \mathrm{min}$ & $193(60.3 \%)$ \\
\hline$\geq 30,<60 \mathrm{ml} / \mathrm{min}$ & $75(23.4 \%)$ \\
\hline$<30 \mathrm{ml} / \mathrm{min}$ & $52(16.3 \%)$ \\
\hline \multicolumn{2}{|l|}{ Baseline laboratory data, mean $\pm \mathrm{SD}$} \\
\hline Total bilirubin $(\mu \mathrm{mol} / \mathrm{L})$ & $20.31 \pm 49.81$ \\
\hline ALT (U/L) & $74.04 \pm 173.2$ \\
\hline AST (U/L) & $85.37 \pm 217.5$ \\
\hline Total protein $(\mathrm{g} / \mathrm{L})$ & $59.54 \pm 9.66$ \\
\hline Serum albumin $(g / L)$ & $31.6 \pm 5.46$ \\
\hline Platelet count $\left(10^{9} / \mathrm{L}\right)$ & $240.47 \pm 120.65$ \\
\hline Haemoglobin $(\mathrm{g} / \mathrm{L})$ & $109.32 \pm 22.99$ \\
\hline Intensive care, $\mathrm{n}(\%)$ & $168(52.5 \%)$ \\
\hline Shock $^{\mathrm{a}}, \mathrm{n}(\%)$ & $107(33.4 \%)$ \\
\hline \multicolumn{2}{|l|}{ Comorbid diseases, $\mathrm{n}(\%)$} \\
\hline Hypertension & $174(54.4 \%)$ \\
\hline Diabetes & $75(23.4 \%)$ \\
\hline Malignant tumor & $19(5.9 \%)$ \\
\hline Stroke & $93(29.0 \%)$ \\
\hline Cardiac insufficiency & $97(30.3 \%)$ \\
\hline Chronic lung disease & $25(7.8 \%)$ \\
\hline \multicolumn{2}{|l|}{ Concurrent medications, $\mathrm{n}(\%)$} \\
\hline Aspirin & $45(14.1 \%)$ \\
\hline Clopidogrel & $38(11.8 \%)$ \\
\hline Low molecular weight heparins & $79(24.7 \%)$ \\
\hline
\end{tabular}

$A L T$ alanine aminotransferase; $A S T$ glutamic oxaloacetic transaminase

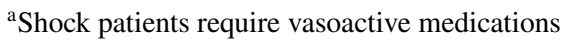

was $24.57 \pm 15.58$ days and linezolid therapy duration was $7.40 \pm 5.75$ days. (Table 1 ).

\section{Comparison of the clinical characteristics between thrombocytopenia group and non thrombocytopenia group}

The thrombocytopenia patients were classified into 73 cases (22.8\%) and 136 cases (42.5\%) defined by criterion 1 and 2, respectively. Among the 320 patients included in this study, 18 patients stopped linezolid due to severe thrombocytopenia, of which, the platelet count of 16 patients dropped to $25-49 \times 10^{9} / \mathrm{L}$, and two patients dropped to less than $25 \times 10^{9} / \mathrm{L}$. Table 2 compared the clinical characteristics between thrombocytopenia group and non thrombocytopenia group including sex, age, hospitalization duration, bilirubin level, creatinine clearance, intensive care, shock, linezolid therapy duration, comorbidities and concurrent medications. Patients were divided into three groups according to creatinine clearance rate $(\geq 60 \mathrm{~mL} / \mathrm{min}, \geq 30$ and $<60 \mathrm{~mL} /$ $\mathrm{min}$, and $<30 \mathrm{~mL} / \mathrm{min}$ ), and also according to linezolid therapy duration ( $<7$ days, $\geq 7$ and $<14$ days, and $\geq 14$ days). The independent-samples t-test and Pearson's chi-square test showed that there were significant differences in age $(p=0.015)$, baseline platelet count $(p<0.001)$, creatinine clearance $(p=0.005)$, shock $(p=0.007)$, linezolid therapy duration $(p=0.002)$ and cardiac insufficiency $(p=0.004)$ between thrombocytopenia group and non thrombocytopenia group( criterion-1); the analysis also indicated significant differences for age ( $p=0.026)$, hospitalization days $(p=0.024)$, shock $(p=0.012)$ and linezolid therapy duration $(p<0.001)$ between thrombocytopenia group and non thrombocytopenia group( criterion-2) (Table 2).

\section{Risk factors for linezolid-induced thrombocytopenia}

Age, sex, creatinine clearance, baseline platelet count, linezolid therapy duration and shock were analyzed as covariates in binary logistic regression model, with results indicating that criterion-1 thrombocytopenia was associated with baseline platelet count $(\mathrm{OR}=0.992,95 \% \mathrm{CI}=0.988-0.995$, $p<0.001$ ), linezolid therapy duration $(\geq 7$ and $<14$ days: $\mathrm{OR}=3.463,95 \% \mathrm{CI}=1.796-6.676, p<0.001 ; \geq 14$ days: $\mathrm{OR}=2.508,95 \% \mathrm{CI}=1.047-6.006, p=0.039)$ and shock $(\mathrm{OR}=2.091,95 \% \mathrm{CI}=1.127-3.880, P=0.019)$, while criterion-2 thrombocytopenia was associated with linezolid therapy duration $(\geq 7$ and $<14$ days: $O R=3.767,95 \%$ $\mathrm{CI}=2.213-6.413, p<0.001 ; \geq 14$ days: $\mathrm{OR}=2.615,95 \%$ $\mathrm{CI}=1.260-5.429, p=0.010)$ and shock $(\mathrm{OR}=1.907,95 \%$ $\mathrm{CI}=1.132-3.213, p=0.015)($ Table 3$)$.

Since shock was a common risk factor for both thrombocytopenia definitions, the cumulative incidence of thrombocytopenia was compared in patients with and without shock using a log-rank test and presented as Kaplan-Meier curves. Kaplan-Meier analysis also indicated a higher cumulative thrombocytopenia incidence in shock patients than in those without shock (Fig. 1). The $p$ values of log-rank tests for the different thrombocytopenia definitions (criterion 1, criterion 2 , early-onset criterion- 1 and early-onset criterion-2 thrombocytopenia) were $0.006,0.021,0.001$ and 0.004 , respectively (Fig. 1).

\section{Comparison of shock between early-onset thrombocytopenia and no thrombocytopenia}

Table 4 compared shock patients requiring vasoactive drugs in terms of early-onset thrombocytopenia or no thrombocytopenia. Pearson's chi-square test indicated significant 
Table 2 Comparison of clinical characteristics between thrombocytopenia and no thrombocytopenia

\begin{tabular}{|c|c|c|c|c|c|c|}
\hline \multirow[t]{2}{*}{ Variables } & \multicolumn{3}{|c|}{ Platelet count $<100 \times 10^{9} / \mathrm{L}^{\mathrm{a}}$} & \multicolumn{3}{|c|}{ The drop decrease of platelet count was $\geq 25 \%^{b}$} \\
\hline & No thrombocytopenia & Thrombocytopenia $^{a}$ & $p$ & No thrombocytopenia & Thrombocytopenia $^{\mathrm{a}}$ & $p$ \\
\hline Total, n (\%) & $247(77.2 \%)$ & $73(22.8 \%)$ & & $184(57.5 \%)$ & $136(42.5 \%)$ & \\
\hline Sex, n $(\%)$ & & & 0.302 & & & 0.214 \\
\hline Male & $167(75.6 \%)$ & $54(24.4 \%)$ & & $122(55.2 \%)$ & $99(44.8 \%)$ & \\
\hline Female & $80(80.8 \%)$ & $19(19.2 \%)$ & & $62(62.6 \%)$ & $37(37.4 \%)$ & \\
\hline Age (years) & $68.47 \pm 16.12$ & $73.78 \pm 16.77$ & 0.015 & $67.93 \pm 16.15$ & $72.05 \pm 16.48$ & $\mathbf{0 . 0 2 6}$ \\
\hline Hospitalization days & $24.19 \pm 15.29$ & $25.84 \pm 16.57$ & 0.429 & $22.82 \pm 14.00$ & $26.93 \pm 17.26$ & 0.024 \\
\hline Total bilirubin level & $18.18 \pm 39.06$ & $27.53 \pm 75.58$ & 0.312 & $20.03 \pm 44.94$ & $20.69 \pm 55.91$ & 0.908 \\
\hline ALT & $61.69 \pm 154.05$ & $115.84 \pm 222.65$ & 0.055 & $70.92 \pm 175.82$ & $78.26 \pm 170.21$ & 0.709 \\
\hline AST & $70.35 \pm 207.09$ & $136.19 \pm 244.14$ & 0.039 & $81.45 \pm 238.08$ & $90.68 \pm 186.70$ & 0.708 \\
\hline Total protein $(\mathrm{g} / \mathrm{L})$ & $59.51 \pm 9.41$ & $59.64 \pm 10.53$ & 0.923 & $59.39 \pm 9.66$ & $59.75 \pm 9.69$ & 0.740 \\
\hline Serum albumin $(g / L)$ & $31.89 \pm 5.51$ & $30.77 \pm 5.23$ & 0.125 & $32.13 \pm 5.40$ & $30.96 \pm 5.48$ & 0.059 \\
\hline Haemoglobin (g/L) & $108.03 \pm 23.00$ & $110.95 \pm 27.72$ & 0.366 & $108.48 \pm 23.80$ & $109.00 \pm 24.69$ & 0.848 \\
\hline Platelet count $\left(10^{9} / \mathrm{L}\right)$ & $236.45 . \pm 118.15$ & $162.12 \pm 81.53$ & 0.000 & $214.66 \pm 113.35$ & $225.88 \pm 117.42$ & 0.390 \\
\hline Creatinine clearance, $\mathrm{mL} / \mathrm{min}$ & & & 0.005 & & & 0.113 \\
\hline$\geq 60$ & $159(82.4 \%)$ & $34(17.6 \%)$ & & $120(62.2 \%)$ & $73(37.8 \%)$ & \\
\hline$\geq 30,<60$ & $56(74.7 \%)$ & $19(25.3 \%)$ & & $38(50.7 \%)$ & $37(49.3 \%)$ & \\
\hline$<30$ & $32(61.5 \%)$ & $20(38.5 \%)$ & & $26(50.0 \%)$ & $26(50.0 \%)$ & \\
\hline Intensive care, $\mathrm{n}(\%)$ & & & 0.327 & & & 0.205 \\
\hline No intensive care & $121(79.6 \%)$ & $31(20.4 \%)$ & & $93(61.2 \%)$ & $59(38.8 \%)$ & \\
\hline Intensive care & $126(75.0 \%)$ & $42(25.0 \%)$ & & $91(54.2 \%)$ & $77(45.8 \%)$ & \\
\hline Shock $^{\mathrm{c}}, \mathrm{n}(\%)$ & & & 0.007 & & & 0.012 \\
\hline No shock & $174(81.7 \%)$ & $39(18.3 \%)$ & & $133(62.4 \%)$ & $80(37.6 \%)$ & \\
\hline Shock & $73(68.2 \%)$ & $34(31.8 \%)$ & & $51(47.7 \%)$ & $56(52.3 \%)$ & \\
\hline $\begin{array}{l}\text { Linezolid therapy duration days, } \\
\text { mean } \pm \text { SD }\end{array}$ & $6.93 \pm 5.47$ & $9.01 \pm 6.41$ & 0.006 & $6.93 \pm 5.47$ & $9.01 \pm 6.41$ & 0.006 \\
\hline Linezolid therapy duration, days, & & & 0.002 & & & 0.000 \\
\hline$<7 d$ & $145(84.8 \%)$ & $26(15.2 \%)$ & & $121(70.7 \%)$ & $50(29.3 \%)$ & \\
\hline$\geq 7,<14 \mathrm{~d}$ & $74(67.9 \%)$ & $35(32.1 \%)$ & & $45(41.3 \%)$ & $64(58.7 \%)$ & \\
\hline$\geq 14 d$ & $28(70.0 \%)$ & $12(30.0 \%)$ & & $18(45.0 \%)$ & $22(55.0 \%)$ & \\
\hline \multicolumn{7}{|l|}{ Comorbid diseases, $n(\%)$} \\
\hline Malignant tumor & $15(78.9 \%)$ & $4(21.1 \%)$ & 0.850 & $12(63.2 \%)$ & $7(36.8 \%)$ & 0.607 \\
\hline Hypertension & $135(77.6 \%)$ & $39(22.4 \%)$ & 0.853 & $102(58.6 \%)$ & $72(41.4 \%)$ & 0.658 \\
\hline Cardiac insufficiency & $65(67.0 \%)$ & $32(33.0 \%)$ & 0.004 & $48(49.5 \%)$ & $49(50.5 \%)$ & 0.056 \\
\hline Diabetes & $58(77.3 \%)$ & $17(22.7 \%)$ & 0.973 & $48(64.0 \%)$ & $27(36.0 \%)$ & 0.193 \\
\hline Stroke & $77(82.8 \%)$ & $16(17.2 \%)$ & 0.126 & $58(62.4 \%)$ & $35(37.6 \%)$ & 0.260 \\
\hline Chronic lung disease & $18(72.0 \%)$ & $7(28.0 \%)$ & 0.520 & $15(60.0 \%)$ & $10(40.0 \%)$ & 0.792 \\
\hline \multicolumn{7}{|l|}{ Concurrent medications } \\
\hline Aspirin & $24(53.3 \%)$ & $21(46.7 \%)$ & 0.542 & $23(51.1 \%)$ & $22(48.9 \%)$ & 0.541 \\
\hline Clopidogrel & $32(84.2 \%)$ & $6(15.8 \%)$ & 0.272 & $26(68.4 \%)$ & $12(31.6 \%)$ & 0.147 \\
\hline Low molecular weight heparin & $56(70.9 \%)$ & $23(29.1 \%)$ & 0.124 & $42(53.2 \%)$ & $37(46.8 \%)$ & 0.369 \\
\hline
\end{tabular}

ALT Alanine aminotransferase, AST Glutamic oxaloacetic transaminase

Bold values show statistical significance of differences

${ }^{a}$ Thrombocytopenia with platelet count $<100 \times 10^{9} / \mathrm{L}$ and a decrease in $25 \%$ or more from baseline of the platelet count

${ }^{b}$ Thrombocytopenia due to a platelet count drop decrease of $25 \%$ or more from baseline

${ }^{\mathrm{c}}$ Shock patients require vasoactive medications 
Table 3 Binary logistic regression analysis of determinants associated with thrombocytopenia

\begin{tabular}{|c|c|c|c|c|c|c|}
\hline \multirow[t]{2}{*}{ Variables } & \multicolumn{3}{|c|}{ Platelet count $<100 \times 10^{9} / \mathrm{L}^{\mathrm{a}}$} & \multicolumn{3}{|c|}{$\begin{array}{l}\text { The drop decrease of platelet count } \\
\text { was } \geq 25 \% \text { b }\end{array}$} \\
\hline & OR & $95 \%$ CI & $\mathrm{P}$ & OR & $95 \% \mathrm{CI}$ & $\mathrm{P}$ \\
\hline Age (years) & 1.015 & $0.995-1.035$ & 0.139 & 1.013 & $0.997-1.030$ & 0.105 \\
\hline Sex & 0.631 & $0.324-1.230$ & 0.176 & 0.649 & $0.380-1.108$ & 0.113 \\
\hline \multicolumn{7}{|l|}{$\mathrm{CrCl}, \mathrm{mL} / \mathrm{min}$} \\
\hline$\geq 60$ (reference) & 1.000 & - & - & 1.000 & - & - \\
\hline$\geq 30,<60$ & 0.975 & $0.464-2.048$ & 0.947 & 1.231 & $0.668-2.266$ & 0.505 \\
\hline$<30$ & 1.876 & $0.864-4.071$ & 0.112 & 1.550 & $0.766-3.135$ & 0.223 \\
\hline $\begin{array}{l}\text { Baseline platelet } \\
\text { count }\left(10^{9} / \mathrm{L}\right)\end{array}$ & 0.992 & $0.988-0.995$ & 0.000 & 1.001 & $0.999-1.004$ & 0.201 \\
\hline \multicolumn{7}{|c|}{ Linezolid therapy duration, days, } \\
\hline$<7$ & 1.000 & - & - & 1.000 & - & - \\
\hline$\geq 7,<14$ & 3.463 & $1.796-6.676$ & 0.000 & 3.767 & $2.213-6.413$ & $\mathbf{0 . 0 0 0}$ \\
\hline$\geq 14$ & 2.508 & $1.047-6.006$ & $\mathbf{0 . 0 3 9}$ & 2.615 & $1.260-5.429$ & $\mathbf{0 . 0 1 0}$ \\
\hline Shock $^{\mathrm{c}}$ & 2.091 & $1.127-3.880$ & 0.019 & 1.907 & $1.132-3.213$ & 0.015 \\
\hline
\end{tabular}

$\mathrm{CrCL}$ creatinine clearance rate

Bold values show statistical significance of differences

${ }^{a}$ Thrombocytopenia with platelet count $<100 \times 10^{9} / \mathrm{L}$ and a decrease in $25 \%$ or more from baseline of the platelet count

${ }^{\mathrm{b}}$ Thrombocytopenia due to a platelet count drop decrease of $25 \%$ or more from baseline

${ }^{\mathrm{c}}$ Shock patients require vasoactive medications differences for shock between early-onset criterion-1 thrombocytopenia and no thrombocytopenia $(P=0.001)$, and between early-onset criterion-2 thrombocytopenia and no thrombocytopenia $(p=0.005)$.

\section{Correlation between shock and early-onset thrombocytopenia}

We analyzed the correlation between shock and early-onset thrombocytopenia after adjusting for the confounding factors of age, hospitalization days, creatinine clearance rate and baseline platelet count in the regression model. Binary logistic regression analysis indicated a significant correlation between shock and early-onset criterion-1 thrombocytopenia (adjusted $\mathrm{OR}=2.709,95 \% \mathrm{CI}=1.359-5.398, p=0.005$ ), and early-onset criterion-2 thrombocytopenia (adjusted $\mathrm{OR}=1.900,95 \% \mathrm{CI}=1.113-3.243, p=0.019)$ after eliminating the confounding factors (Table 5).

\section{Discussion}

This study indicated that thrombocytopenia incidence and risk factors differed according to its definition. The criterion-1 thrombocytopenia incidence was $22.8 \%$, baseline platelet count, linezolid therapy duration and shock were independent risk factors. The criterion-2 thrombocytopenia incidence was $42.5 \%$, linezolid therapy duration and shock were independent risk factors. Linezolid therapy duration and shock were common causes of thrombocytopenia regardless of the definition. Kaplan-Meier analysis also indicated that the cumulative thrombocytopenia incidence was higher in shock patients. The binary logistic regression analysis indicated a significant correlation between shock and early-onset thrombocytopenia. As far as we know, this is the first study of that has elucidated the relationship between shock and thrombocytopenia risk.

A previous study that included only intensive-care unit (ICU) patients found a thrombocytopenia incidence of 48.3\% [16], which was higher than in other studies involving patients in general ward. However, there was no difference in thrombocytopenia incidence between ICU patients and non-ICU patients in our study, and shock was indicated as an independent risk factor. Immunosuppression occurs both early and late in the shock response. Patients who survive septic shock often have prolonged clinical trajectories and exhibit chronic immune suppression [17, 18]. The mechanisms of linezolid-induced thrombocytopenia may be related to myelosuppression and immune-mediated platelet destruction [19-21]. In clinical practice, patients with shock requiring vasoactive medications often develop thrombocytopenia after only 1 or 2 days of linezolid treatment, and recover gradually after linezolid discontinuation. Onset and recovery times are consistent with immune-mediated druginduced thrombocytopenia $[19,20]$. In the present study, 24 


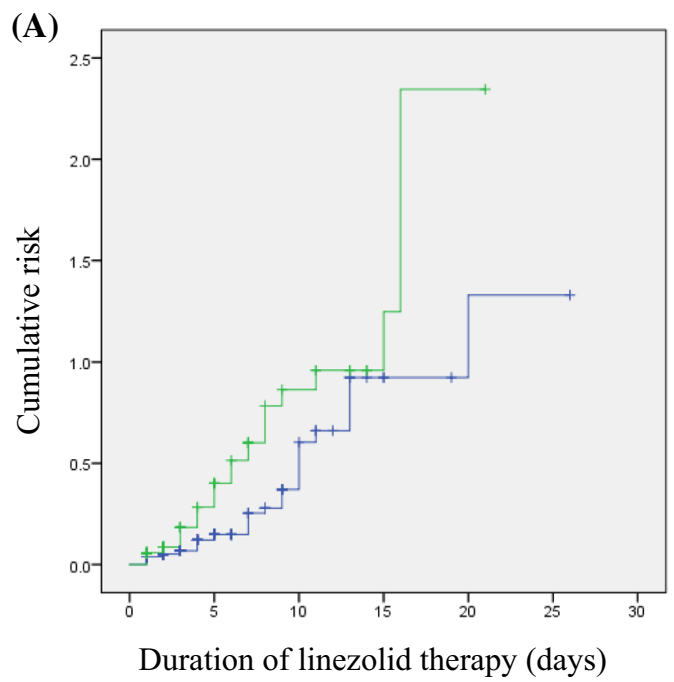

patients with shock

patients without shock

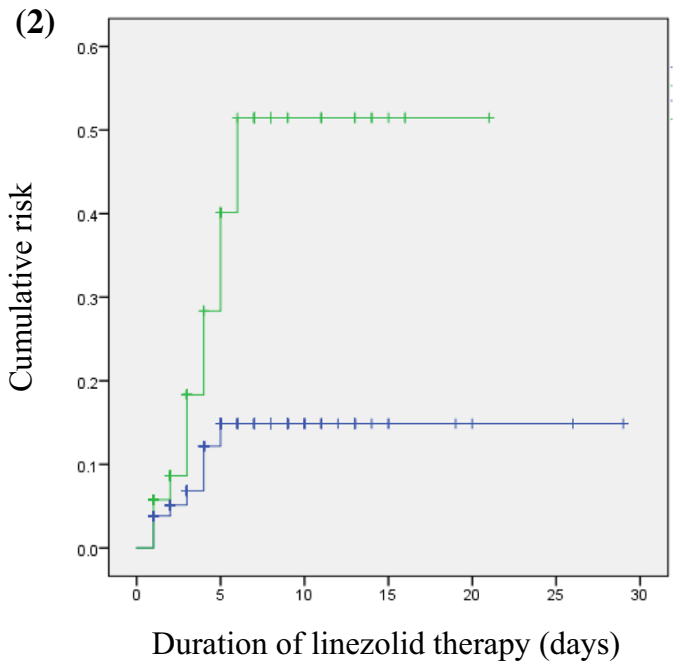

1. B

2. $\mathrm{C}$

3. D patients with shock patients without shock
Fig. 1 Kaplan-Meier estimates of the cumulative incidence of linezolid-induced thrombocytopenia in patients with shock and patients without shock. a Criterion-1thrombocytopenia: thrombocytopenia with platelet count $<100 \times 10^{9} / \mathrm{L}$ and a decrease in $25 \%$ or more from baseline of the platelet count; b Criterion-2 thrombocytopenia: thrombocytopenia due to a platelet count drop decrease of $25 \%$

cases $(22.4 \%)$ and 37 cases (34.6\%) shock patients developed early-onset thrombocytopenia according to criterion 1 and criterion 2 , respectively. The binary logistic regression
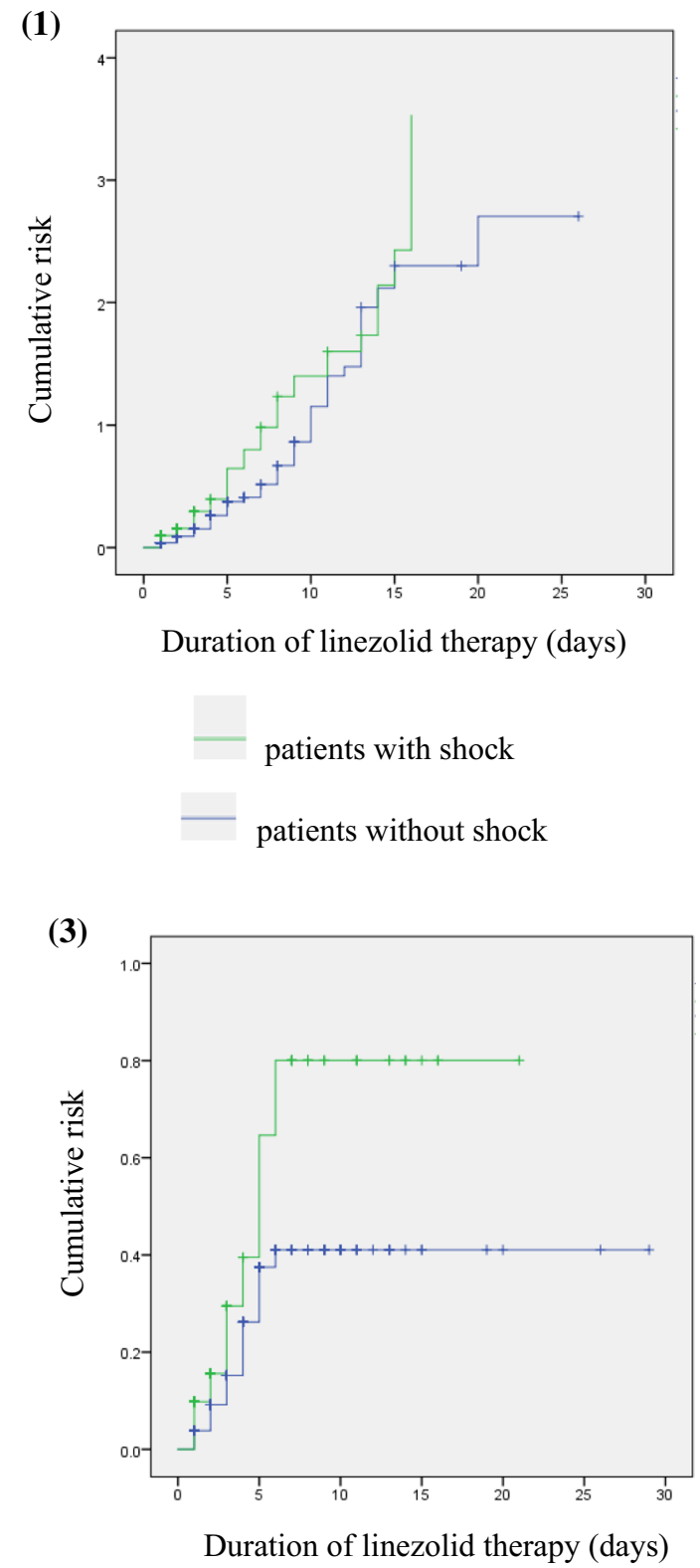
criterion-1 thrombocytopenia occurrence within 6 days of linezolid treatment initiation; d Early-onset criterion-2 thrombocytopenia: criterion- 2 thrombocytopenia occurrence within 6 days of initiating linezolid therapy

analysis indicated a significant correlation between shock and early-onset thrombocytopenia.

Baseline platelet count has been reported as a risk factor for thrombocytopenia. Our study indicated that the risk 
Table 4 Comparison of shock between early-onset thrombocytopenia and no thrombocytopenia

\begin{tabular}{|c|c|c|c|c|c|c|}
\hline & \multicolumn{3}{|c|}{ Platelet count $<100 \times 10^{9} / \mathrm{L}^{\mathrm{a}}$} & \multicolumn{3}{|c|}{ The drop decrease of platelet count was $\geq 25 \%^{b}$} \\
\hline & No thrombocytopenia & $\begin{array}{l}\text { Early-onset } \\
\text { thrombocytopenia }^{\mathrm{c}}\end{array}$ & $p$ & No thrombocytopenia & $\begin{array}{l}\text { Early-onset } \\
\text { thrombocytopenia }^{c}\end{array}$ & $p$ \\
\hline Shock $^{\mathrm{d}}, \mathrm{n}(\%)$ & & & 0.001 & & & 0.005 \\
\hline No shock & $194(91.1 \%)$ & $19(8.9 \%)$ & & $170(79.8 \%)$ & $43(20.2 \%)$ & \\
\hline Shock & $83(77.6 \%)$ & $24(22.4 \%)$ & & $70(65.4 \%)$ & $37(34.6 \%)$ & \\
\hline
\end{tabular}

Bold values show statistical significance of differences

${ }^{a}$ Thrombocytopenia with platelet count $<100 \times 10^{9} / \mathrm{L}$ and a decrease in $25 \%$ or more from baseline of the platelet count

${ }^{\mathrm{b}}$ Thrombocytopenia due to a platelet count drop decrease of $25 \%$ or more from baseline

${ }^{c}$ Early-onset thrombocytopenia: thrombocytopenia occurrence within 6 days of linezolid treatment initiation

${ }^{\mathrm{d}}$ Shock patients require vasoactive medications

Table 5 Binary logistic regression analysis: the correlation between shock ${ }^{\mathrm{a}}$ and early-onset thrombocytopenia

\begin{tabular}{|c|c|c|c|c|c|c|}
\hline \multirow[t]{2}{*}{ Variables } & \multicolumn{3}{|c|}{$\begin{array}{l}\text { Early-onset thrombocytopenia }{ }^{\mathrm{b}} \text { (platelet } \\
\text { count }<100 \times 10^{9} / \mathrm{L}^{\mathrm{c}} \text { ) }\end{array}$} & \multicolumn{3}{|c|}{$\begin{array}{l}\text { Early-onset thrombocytopenia (the drop } \\
\text { decrease of platelet count was } \geq 25 \%^{\mathrm{d}} \text { ) }\end{array}$} \\
\hline & OR & $95 \% \mathrm{CI}$ & $\mathrm{P}$ & OR & $95 \% \mathrm{CI}$ & $\mathrm{P}$ \\
\hline Crude & 2.952 & $1.534-5.681$ & 0.001 & 2.090 & $1.242-3.516$ & 0.005 \\
\hline Adjusted $^{\mathrm{e}}$ & 2.709 & $1.359-5.398$ & 0.005 & 1.900 & $1.113-3.243$ & 0.019 \\
\hline \multicolumn{7}{|c|}{ Bold values show statistical significance of differences } \\
\hline \multicolumn{7}{|c|}{ a Shock patients require vasoactive medications } \\
\hline \multicolumn{7}{|c|}{$\begin{array}{l}\text { bEarly-onset thrombocytopenia: thrombocytopenia occurrence within } 6 \text { days of linezolid treatment initia- } \\
\text { tion }\end{array}$} \\
\hline \multicolumn{7}{|c|}{$\begin{array}{l}\text { c Thrombocytopenia with platelet count }<100 \times 10^{9} / \mathrm{L} \text { and a decrease in } 25 \% \text { or more from baseline of the } \\
\text { platelet count }\end{array}$} \\
\hline \multicolumn{7}{|c|}{${ }^{\mathrm{d}}$ Thrombocytopenia due to a platelet count drop decrease of $25 \%$ or more from baseline } \\
\hline
\end{tabular}

factors for linezolid-induced thrombocytopenia differed according to its definition. Baseline platelet count was an independent risk factor when thrombocytopenia was defined as a platelet count of $<100 \times 10^{9} / \mathrm{L}$ (whether patients with baseline platelet counts $<100 \times 10^{9} / \mathrm{L}$ were excluded or not), but not when it was defined as a decrease of $\geq 25 \%$ in baseline platelet count, which was consistent with previous reports [6-15]. There is currently no unified diagnostic criterion for linezolid-induced thrombocytopenia. In some previous studies, thrombocytopenia was defined as a platelet count of $<100 \times 10^{9} / \mathrm{L}$ after treatment (patients with baseline platelet counts $<100 \times 10^{9} / \mathrm{L}$ were excluded in some studies), while in others thrombocytopenia was defined as a decrease of $\geq 25 \%$ or $30 \%$ in baseline platelet count. According to the drug-related thrombocytopenia criteria adopted by the University of Oklahoma Health Sciences Center [22], when platelet counts are lower than the lower limit of normal $\left(<100 \times 10^{9} / \mathrm{L}\right)$ after treatment, it is considered thrombocytopenia. This criterion better reflects platelet counts after linezolid treatment to indicate severe linezolid-induced thrombocytopenia, but it includes some patients with small decreases in platelet counts. For example, the baseline platelet count of some patients is $101 \times 10^{9} / \mathrm{L}$, and the platelet count is $98 \times 10^{9} / \mathrm{L}$ after treatment (which is defined as thrombocytopenia), but this decrease has no clinical significance, which will result in an artificially higher thrombocytopenia incidence. We therefore defined thrombocytopenia as either a platelet count of $<100 \times 10^{9} / \mathrm{L}$ and a decrease in $25 \%$ or more from baseline of the platelet count, or a platelet count drop decrease of $25 \%$ or more from baseline.

Patients included in this study were divided into three groups according to creatinine clearance rate $(\geq 60 \mathrm{~mL} /$ $\mathrm{min}, \geq 30$ and $<60 \mathrm{~mL} / \mathrm{min}$, and $<30 \mathrm{~mL} / \mathrm{min}$ ), and Pearson's chi-square test results indicated a significant difference in creatinine clearance rate between patients with criterion-1 thrombocytopenia and patients without thrombocytopenia. However, binary logistic regression analysis indicated that the creatinine clearance rate was not a risk factor for criterion-1 thrombocytopenia, which was not consistent with previous reports [6-13]. Such differences may be at least partially attributable to the inclusion of patients from different racial groups, different clinical conditions and different 
definitions of thrombocytopenia. A standard definition is necessary to facilitate comparisons between studies.

Linezolid treatment duration was an independent risk factor for thrombocytopenia, which is consistent with previous reports. The recommended duration for linezolid treatment is 10-14 days for pneumonia and skin and softtissue infections, and 14-28 days for vancomycin-resistant enterococci. Gerson et al. [23] reported that thrombocytopenia was evident after $>2$ weeks of linezolid treatment. Our study indicated that the relative risks of thrombocytopenia among patients receiving linezolid therapy for 7-14 days were 3.463 and 3.767 times higher than in patients receiving linezolid therapy for $<7$ days for criterion- 1 and -2 thrombocytopenia, respectively, and among patients receiving linezolid therapy for $\geq 14$ days were 2.508 and 2.615 times for criterion- 1 and -2 thrombocytopenia, respectively. The increased thrombocytopenia incidence was not proportional to linezolid therapy duration, this is consistent with the literature that thrombocytopenia usually occurs within 10-14 $\mathrm{d}$ after initiation of therapy $[24,25]$, which may be due to the mechanisms of linezolid-induced thrombocytopenia resulting from myelosuppression and immune-mediated platelet destruction. Our findings indicated the necessity for monitoring platelet counts frequently in all patients who received linezolid treatment for longer than 7 days.

Our study had three limitations. Firstly, we could not control for all confounding factors due to the retrospective design of the study. Secondly, patient selection bias may have occurred since the study was only conducted within our hospital, which limits the generalizability of the results. Thirdly, the sample of our study was small. Further multicenter prospective studies with large samples would be required for to ensure adequate statistical power in the analyses. However, our study indicated that shock was a risk factor for thrombocytopenia, which will be useful for establishing an appropriate drug utilization strategy in linezolid therapy.

\section{Conclusions}

This study found that thrombocytopenia incidence and risk factors differed according to its definition. Baseline platelet count was an independent risk factor when thrombocytopenia was defined as a platelet count of $<100 \times 10^{9} / \mathrm{L}$ (whether or not patients with baseline platelet counts $<100 \times 10^{9} / \mathrm{L}$ were excluded), but not when defined as a decrease of $\geq 25 \%$ in baseline platelet count. Linezolid therapy duration and shock patients require vasoactive medications were common causes of thrombocytopenia, regardless of its definition. Shock was correlated with early-onset thrombocytopenia. Platelet count should be monitored during linezolid therapy especially during long-duration linezolid therapy and in patients with shock requiring vasoactive medications.

Acknowledgements We would like to express our appreciation to Information Centre in our hospital, for providing the data utilized in this study. We thank all the staff who contributed to the study.

Authors' contribution XN initiated the study and introduced the research question. All authors participated in designing the study. JW and $\mathrm{XZ}$ performed the data extractions. $\mathrm{XH}$ and $\mathrm{LP}$ conducted the analyses. All authors discussed the results. $\mathrm{XH}$ wrote the manuscript, and all authors reviewed and edited it. All authors accepted the final version of the manuscript.

Funding No specific funding was received.

Data availability The datasets generated during the current study are available from the corresponding author on reasonable request.

Conflicts of interest The authors declare that they have no conflict of interest.

Ethics approval The study design was approved by the ethics review board of Xi'an Central Hospital (No. LW-2021-011).

\section{References}

1. Johnson JR, Lindley D, Paterson A, David L, Pasculle. Linezolid: The first oxazolidinone antimicrobial. Ann Internal Med. 2003;138.

2. Lin AH, Murray RW, Vidmar TJ, Marotti KR. The oxazolidinone eperezolid binds to the 50S ribosomal subunit and competes with binding of chloramphenicol and lincomycin. Antimicrob Agents Chemother. 1997;41:2127-31.

3. Swaney SM, Aoki H, Ganoza MC, Shinabarger DL. The oxazolidinone linezolid inhibits initiation of protein synthesis in bacteria. Antimicrob Agents Chemother. 1998;42:3251.

4. Tang S, Yao L, Hao X, Zhang X, Liu G, Liu X, et al. Efficacy, safety and tolerability of linezolid for the treatment of XDR-TB: a study in China. Eur Respirat J. 2016;45.

5. Organization W H. WHO treatment guidelines for drug-resistant tuberculosis 2016 update. 2016.

6. Choi GW, Lee J-Y, Chang MJ, Kim YK, Cho Y, Yu YM, et al. Risk factors for linezolid-induced thrombocytopenia in patients without haemato-oncologic diseases. Basic Clin Pharmacol. 2019; 124:228-34.

7. Hanai Y, Matsuo K, Ogawa M, Higashi A, Kimura I, Hirayama $\mathrm{S}$, et al. A retrospective study of the risk factors for linezolidinduced thrombocytopenia and anemia. J Infect Chemother. 2016;22:536-42.

8. Chen C, Guo D-H, Cao X, Cai Y, Xu Y, Zhu M, et al. Risk factors for thrombocytopenia in adult chinese patients receiving linezolid therapy. Curr Ther Res Clin E. 2012;73:195-206.

9. Hirano R, Sakamoto Y, Tachibana N, Ohnishi M. Retrospective analysis of the risk factors for linezolid-induced thrombocytopenia in adult Japanese patients. Int J Clin Pharm-net. 2014;36:795-9.

10. Takahashi Y, Takesue Y, Nakajima K, Ichiki K, Tsuchida T, Tatsumi S, et al. Risk factors associated with the development of thrombocytopenia in patients who received linezolid therapy. $\mathbf{J}$ Infect Chemother. 2011;17:382-7. 
11. Kaya Kılıç E, Bulut C, Sönmezer MÇ, Ozel Ö, Hatipoğlu CA, Ertemet GT, et al. Risk factors for linezolid-associated thrombocytopenia and negative effect of carbapenem combination. J Infect Dev Countr. 2019;13:886-91.

12. Natsumoto B, Yokota K, Omata F, Furukawa Ke. Risk factors for linezolid-associated thrombocytopenia in adult patients. Infection. 2014;42:1007-12.

13. Sato Y, Iguchi M, Kato Y, Morioka H, Hirabayashi A, Tetsuka $\mathrm{N}$, et al. Number of concomitant drugs with thrombocytopenic adverse effects and the extent inflammatory response resolution are risk factors for thrombocytopenia in patients treated with linezolid for more than 14 days. Nagoya J Med Sci. 2020;82:407-14.

14. Bi L-Q, Zhou J, Huang M, Zhou S-M. Efficacy of linezolid on gram-positive bacterial infection in elderly patients and the risk factors associated with thrombocytopenia. Pak J Med Sci. 2013;29:837-42.

15. Niwa T, Watanabe T, Suzuki A, Ohmri T, Tsuchiya M, Suzuki T, et al. Reduction of linezolid-associated thrombocytopenia by the dose adjustment based on the risk factors such as basal platelet count and body weight. Diagn Micr Infec Dis. 2014;79:93-7.

16. Kim H-S, Lee E, Cho Y-J, Lee Y-J, Rhie S-J. Linezolid-induced thrombocytopenia increases mortality risk in intensive care unit patients, a 10 year retrospective study. J Clin Pharm Ther. 2019;44:84-90.

17. Hotchkiss RS, Moldawer LL, Opal SM, Reinhart K, Turnbull I, Vincent JL. Sepsis and septic shock. Nat Rev Dis Primers. 2016; 2.

18. Annane D, Bellissant E, Cavaillon J-M. Septic Shock Lancet. 2005;365:63-78.
19. Aster RH, Bougie DW. Drug-induced immune thrombocytopenia. Drug Saf. 2007;27:1243-52.

20. Danese E, Montagnana M, Favaloro EJ, Lippi G. Drug-induced thrombocytopenia: mechanisms and laboratory diagnostics. Semin Thromb Hemost. 2019;46:264-74.

21. Bernstein WB, Trotta RF, Rector JT, Tjaden JA, Barile AJ. Mechanisms for linezolid-induced anemia and thrombocytopenia. Ann Pharmacother. 2003;37:517.

22. University of Oklahoma Health Sciences Center [EB/OL]. [06.07.2007]. https://moon.Ouhsc.edu/jgeorge/ditp_criteria.htm.

23. Gerson SL, Kaplan SL, Bruss JB, Le V, Arellano FM, Hafkin B, et al. Hematologic effects of linezolid: summary of clinical experience. Antimicrob Agents Ch. 2002;46:2723-6.

24. Nasraway SA, Shorr AF, Kuter DJ, Naomi O, Cammarata SK. Linezolid does not increase the risk of thrombocytopenia inpatients with nosocomial pneumonia: comparative analysis of linezolid and vancomycin use. Clin Infect Dis. 2003;37:1609-16.

25. Minson Q, Gentry CA. Analysis of linezolid-associated hematologic toxicities in a large veterans affairs medical center. Pharmacotherapy. 2010;30:895-903.

Publisher's Note Springer Nature remains neutral with regard to jurisdictional claims in published maps and institutional affiliations. 